\title{
Trigeminal neuralgia caused by brain arteriovenous malformations: A case report and literature review
}

\author{
YONGJIE YUAN $^{1}$, YANDONG ZHANG ${ }^{2}$, QI LUO ${ }^{1}$ and JINLU YU ${ }^{1}$ \\ Departments of ${ }^{1}$ Neurosurgery and ${ }^{2}$ Medicine, First Hospital of Jilin University, Changchun, Jilin 130021, P.R. China
}

Received January 17, 2015; Accepted March 1, 2016

DOI: $10.3892 /$ etm.2016.3277

\begin{abstract}
Few cases of trigeminal neuralgia (TGN) induced by brain arteriovenous malformations (bAVMs) have previously been reported. The present case report described one case of TGN caused by bAVMs in a 32-year-old male patient who suffered from recurrent pain in his right cheek for a period of two years, for whom the seizure frequency and duration of pain increased for 6 months. Magnetic resonance imaging was performed, which demonstrated flow-void signals in the abnormal vessels in the right cerebellopontine angle. Subsequent digital subtraction angiography confirmed the diagnosis of bAVMs, and showed the nidus was fed by the right superior cerebellar and the right anterior inferior cerebellar, and drained into the adjacent venous sinuses on the same side. The patient underwent an interventional embolization treatment. TGN was completely relieved following embolization of the majority of the bAVMs. Pain relief may be associated with blocking of the pulsatile compression of the feeding arteries of the bAVMs, the arterialized draining veins or the malformed niduses following embolization, which is similar to the effects induced by microvascular decompression surgery of the trigeminal nerve. In the present case study and review, the underlying mechanism and treatment strategy of TGN caused by bAVMs were discussed in the context of present case, and a literature review was carried out.
\end{abstract}

\section{Introduction}

Trigeminal neuralgia (TGN) is a transient, recurrent and intense pain in the area on the face where the trigeminal (TG) nerve is distributed (1-3). Previous studies have demonstrated that $80-90 \%$ of primary TGN cases are induced by vascular compression of the TG nerve at the root entry zone (REZ) $(1,4)$.

Correspondence to: Dr Jinlu Yu or Dr Qi Luo, Department of Neurosurgery, First Hospital of Jilin University, 71 Xinmin Avenue, Changchun, Jilin 130021, P.R. China

E-mail: jinluyu@hotmail.com

E-mail: LuoQiJilin@hotmail.com

Key words: brain arteriovenous malformation, trigeminal neuralgia, treatment
Superior cerebellar artery (SCA) and the anterior inferior cerebellar artery (AICA) are the predominant vessels responsible for the compression, followed by venous vessels $(5,6)$. Brain arteriovenous malformations (bAVMs) often manifest as cerebral hemorrhage, particularly in young patients; therefore, patients with unruptured bAVMs have a 2-4\% risk of rupture and bleeding each year, and each bleeding event is associated with an $\sim 18 \%$ risk of mortality $(7,8)$. In addition, bAVMs also manifest as chronic headaches, epilepsy and neurological deficits (8-11). However, there have been relatively few reports of cases of TGN caused by bAVM $(4,12)$. The present case report describes a case of TGN caused by bAVMs in the cerebellopontine angle (CPA). Facial pain was successfully relieved after the patient received partial embolization treatment, which may be associated with the blockade of the pulsatile compression of the feeding arteries, the arterialized draining veins or the malformed niduses. Therefore, TGN caused by bAVMs is a vascular nerve compression disease. In the present case study and review, the underlying mechanism and treatment strategy of TGN caused by bAVMs were discussed in the context of present case, and a literature review was carried out.

\section{Case report}

A 32-year-old male was admitted to the First Hospital of Jilin University (Changchun, China) on December 6, 2014, suffering from recurrent pain in his right cheek for two years, which had increased in severity over the previous 6 months. The patient reported that the pain began three years previously as a stabbing-like, intense pain that occurred suddenly on the right lower lip and mandible without any prior signs; each episode of pain lasted for 10-30 sec. The first period of pain lasted $\sim 1$ week and was self-relieved without medication. The pain recurred following a period of 6 months, and the duration of each episode of pain extended to 2-3 min. The patient was administered $800 \mathrm{mg}$ oral carbamazepine (Novartis, Basel, Switzerland) daily, which was effective at relieving the pain on the right side of the face of the patient. In the 6 months prior to hospital admission, the frequency of the pain in the right cheek of the patient increased. The pain occurred $>6$ times every day and each episode lasted 10-30 min, and daily oral administration of 1,200 mg carbamazepine was not effective at controlling the pain. Clinical examination demonstrated that no significant trigger point could be palpated and there were no abnormalities in corneal reflexes or facial sensation. Subsequent magnetic 
resonance imaging(MRI) demonstrated the presence of flow-void signals in the abnormal vessels in the right CPA (Fig. 1). Digital subtraction angiography (DSA) further confirmed the diagnosis of bAVMs, and demonstrated that the malformed niduses were fed by the right SCA and the AICA, and drained into the adjacent venous sinuses on the same side (Fig. 2). Although the patient only exhibited symptoms of TGN, the patient was treated with interventional embolization, due to the risk that the bAVMs may bleed in the future. Embolization was conducted under general anesthesia with intravenous $0.05-0.1 \mathrm{mg} / \mathrm{kg}$ midazolam (Nhwa, Jiangsu, China), 1.0-1.5 $\mu \mathrm{g} / \mathrm{kg}$ remifentanil (Renfu, Yichang, China), 0.2-0.3 mg/kg etomidate (Nhwa), $0.6 \mathrm{mg} / \mathrm{kg}$ rocuronium (Organon, Brussels, Belgium), and was maintained with $0.6 \mathrm{mg} / \mathrm{kg}$ propofol (Nhwa), $5-10 \mathrm{mg} /(\mathrm{kg} / \mathrm{h}$ ) and 0.1-0.2 $\mu \mathrm{g} /(\mathrm{kg} / \mathrm{min})$ remifentanil (Renfu, Yichang, China). Following arterial puncture and sheath insertion in the femoral artery using the Seldinger technique (13), a 6F-guiding catheter (Cordis Co., Miami, FL, USA) was inserted into the left vertebral artery. Under the guidance of a Mirage micro-wire (ev3, Irvine, California, USA), a Marathon micro-catheter was inserted into the bAVMs. The micro-catheter entered the bAVMs via the right AICA and onyx-18 glue (ev3) was slowly injected. Onyx-18 glue diffused well in the bAVMs and embolized the majority of the malformations. Angiography was conducted immediately following surgery, which demonstrated that the majority of the bAVMs had disappeared and the draining veins were patent (Fig. 2). There were no complications following the surgical procedure and the pain in the right cheek of the patient was completely relieved one week following the surgical procedure. The patient was satisfied with the outcome of the embolization surgery. Post-surgical follow-ups were conducted for a period of two years via telephone. The patient experienced no further pain in his right cheek, and thus chose not to return to the hospital for a follow-up reexamination. The present study was approved by the Ethics Committee of the First Hospital of Jilin University and patient informed consent was obtained prior to the study.

\section{Literature review}

A total of 29 studies $(4,12,14-40)$ which reported TGN caused by bAVMs were retrieved from the PubMed database, the majority of which were clinical reports that described individual cases. We searched the PubMed database up to 2015 using the key words 'AVM', 'bAVM', 'arteriovenous malformation', 'cerebral arteriovenous malformation', 'brain arteriovenous malformation', 'Cerebral vascular malformation' in combination with 'trigeminal neuralgia' and 'TGN'. Abstracts were reviewed to identify relevant literature, only the TGN caused by bAVM related literatures were enrolled.

A total of 40 patients with TGN caused by bAVMs were reported in these 29 studies (Table I; 4,12,14-40). Excluding two patients for whom the information was incomplete $(27,33)$, there were 24 male and 14 female patients (male:female ratio, 1.71) aged between 23 and 69 years with a mean age of $46.8 \pm 14.7$ years. These patients presented with bAVMs located in the CPA $(n=19)(12,16,17,20,23,27-33,36,38)$, cerebellar hemisphere $(n=8)(15,18,21,26,34,35,40)$, the TG nerve $(n=7)$ $(22,25,39)$, the cerebellar vermis $(n=3)(4,14,24)$, the pontine $(n=1)(37)$, the septum pellucidum $(n=1)(19)$ and both the cerebellar hemisphere and cerebellar vermis $(n=1)(38)$ (Fig. 3). Five of these patients had a confirmed history of repeated hemorrhage $(19,29,32,33,35)$. The majority of these patients suffered from TGN on the same side as the bAVM lesions; however, one patient suffered from TGN on the contralateral side of the bAVMs lesions (40), and two patients suffered from coexistent TGN and hemifacial spasm on the same side $(14,16)$. In terms of treatment, 2 patients received drug therapy (17) and 1 patient was without mention of treatment (12). The other 37 patients received non-drug treatment $(4,14-40), 11$ patients received surgical resection alone (19,25,27-30,32), 2 patients received surgical resection following partial embolization $(23,26)$, 1 patient received surgical resection following destructive neurosurgical manipulation of the TG nerve(31), 3 patients received surgical resection of bAVMs combined with microvascular decompression (MVD; 14,15,18,20,24), 5 patients received an interventional embolization treatment, 2 patients received radiotherapy following embolization $(4,21), 6$ patients received MVD treatment alone (37-39), 2 patients received radiotherapy following MVD $(36,40), 4$ patients received destructive neurosurgical manipulation of the TG nerve $(16,17,33,38)$, and 1 patient received stereotactic radiotherapy (SRS; 22) (Fig. 4).

\section{Discussion}

TGN is one of the most common symptoms of neurovascular compression, and is a demyelinating lesion of the sensory fibers of the TG nerve caused by compression of the TG nerve at REZ by the SCA and the AICA (1-4). However, TGN caused by bAVMs remains rare, accounting for $0.22-1.78 \%$ of primary TGN cases $(12,16,38)$. The present case study described a case of TGN caused by bAVMs in a middle-aged male patient suffering from recurrent pain in the right cheek. MRI and DSA analyses demonstrated bAVMs in the CPA of the posterior fossa on the affected side. As it remains difficult to distinguishing the symptoms of TGN caused by bAVMs, particularly TGN induced by insignificant bAVMs, which is usually negative in angiography, the symptoms of primary TGN can be easily misdiagnosed $(12,25)$. However, the majority of patients can be accurately diagnosed through MRI and cerebral angiography $(4,20,21,36)$. Previous studies have demonstrated that combined use of three-dimensional (3D) constructive interference in steady state MRI and 3D time of flight angiography clearly demonstrate the anatomic association between the TG nerve and the surrounding vessels, as well as other neighboring structures $(41,42)$. DSA clearly shows the bAVMs, including the feeding arteries, niduses and draining veins, and determines whether they are associated with aneurysms, which is beneficial for the preoperative evaluation of patients (17). Among the 40 previous case reports $(4,12,14-40), 39$ patients $(97.5 \%)$ presented with bAVMs in the posterior fossa. bAVMs located in the posterior fossa are more likely to result in TGN (4,12,14-18,20-40). García-Pastor (38) conducted a study on 375 cases of bAVMs, which demonstrated that $1.3 \%$ of the patients with bAVMs developed TGN, whereas $9.8 \%$ of the patients with bAVMs in the posterior fossa developed TGN. The majority of the patients suffered from TGN on the same side as the bAVMs, and only one patient suffered from TGN on the contralateral side of the lesions, which was predominantly caused by the 


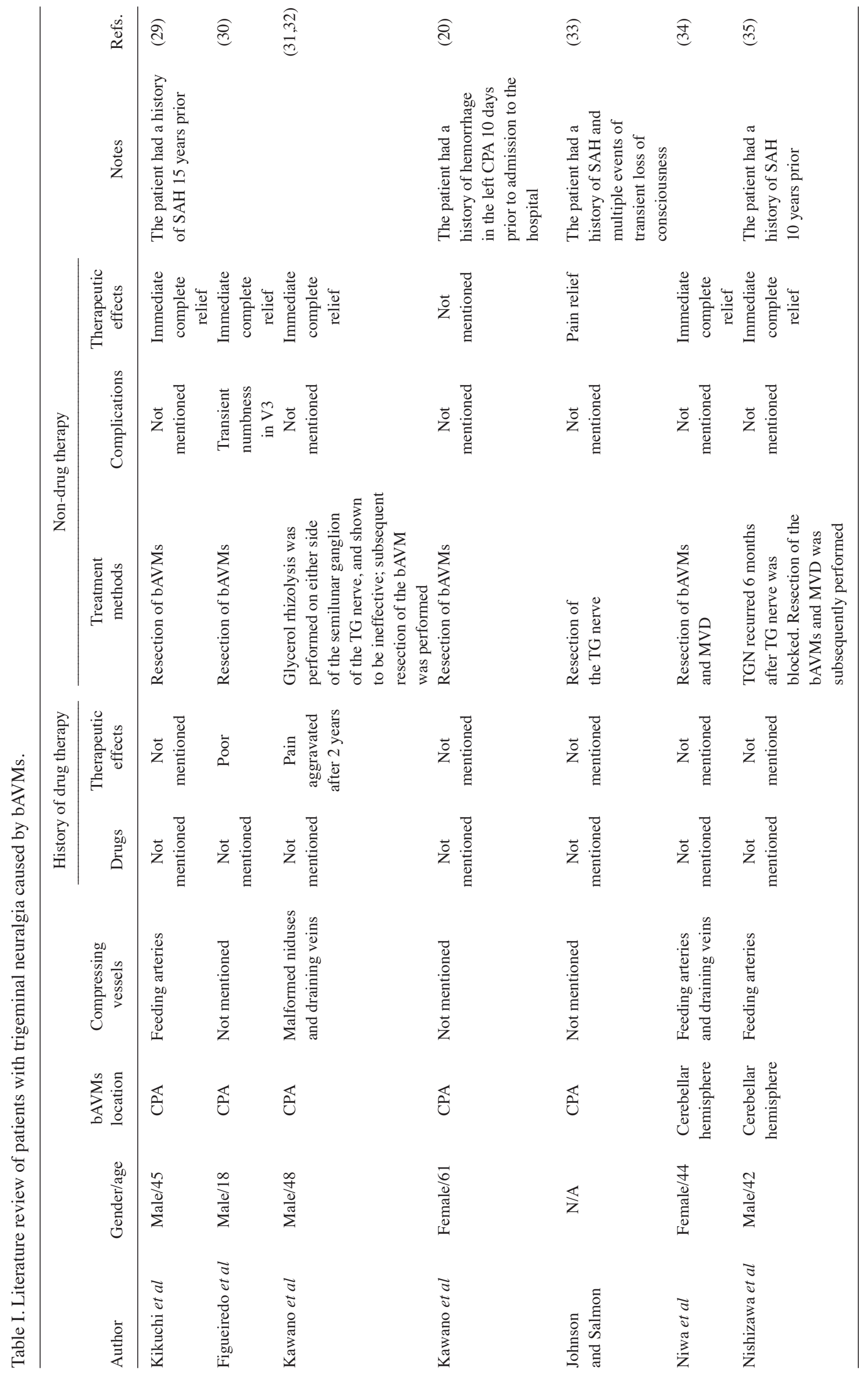




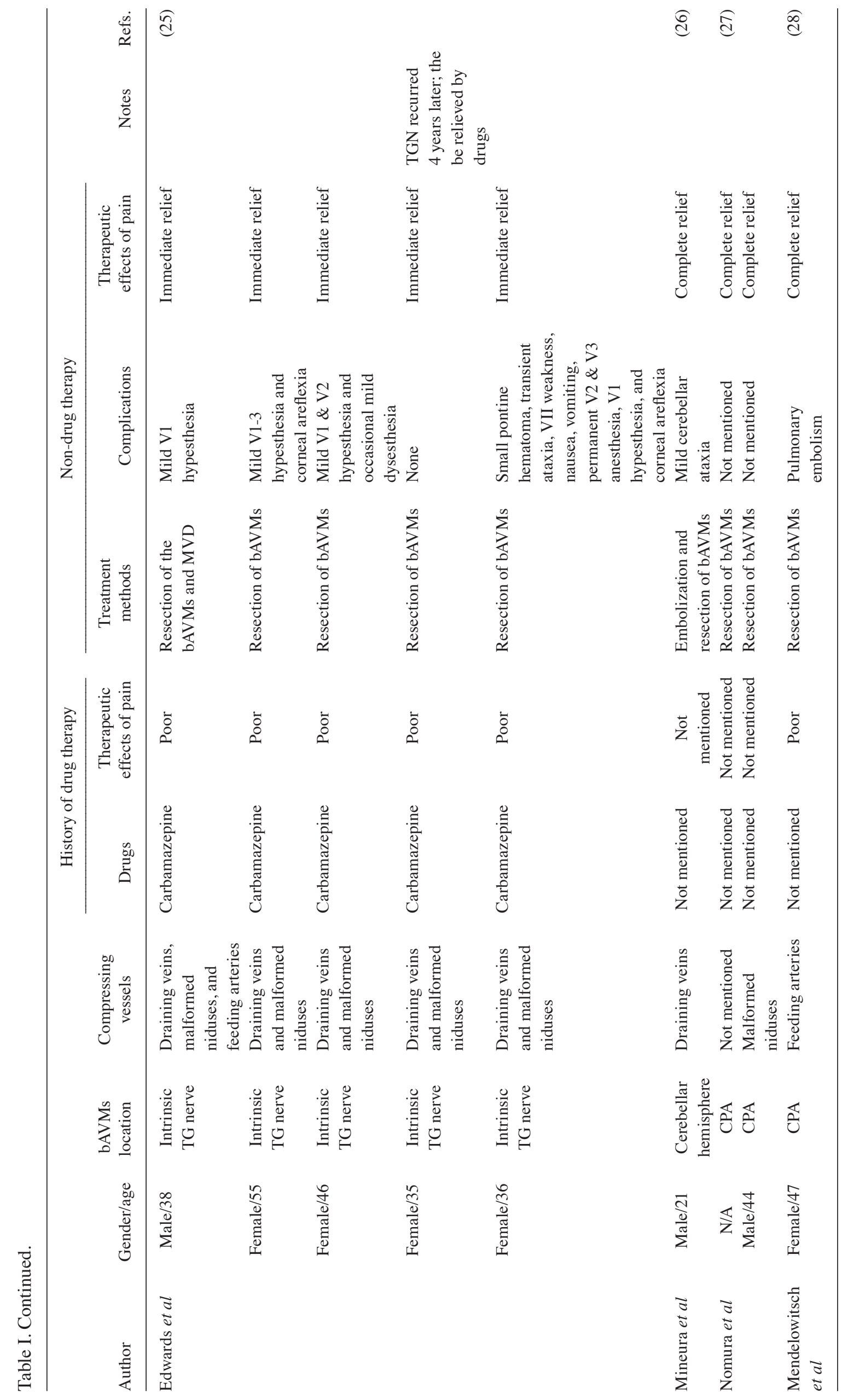




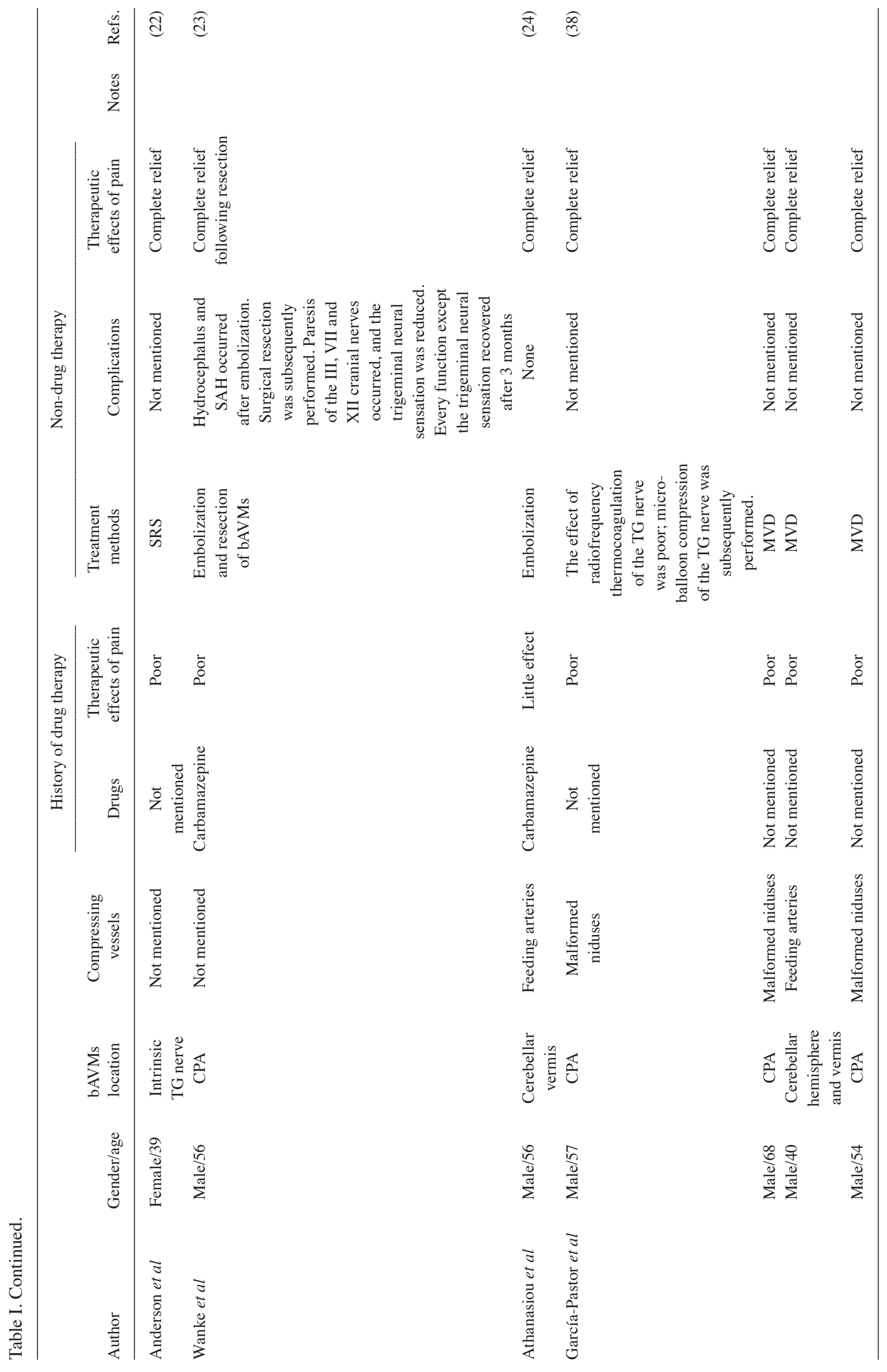




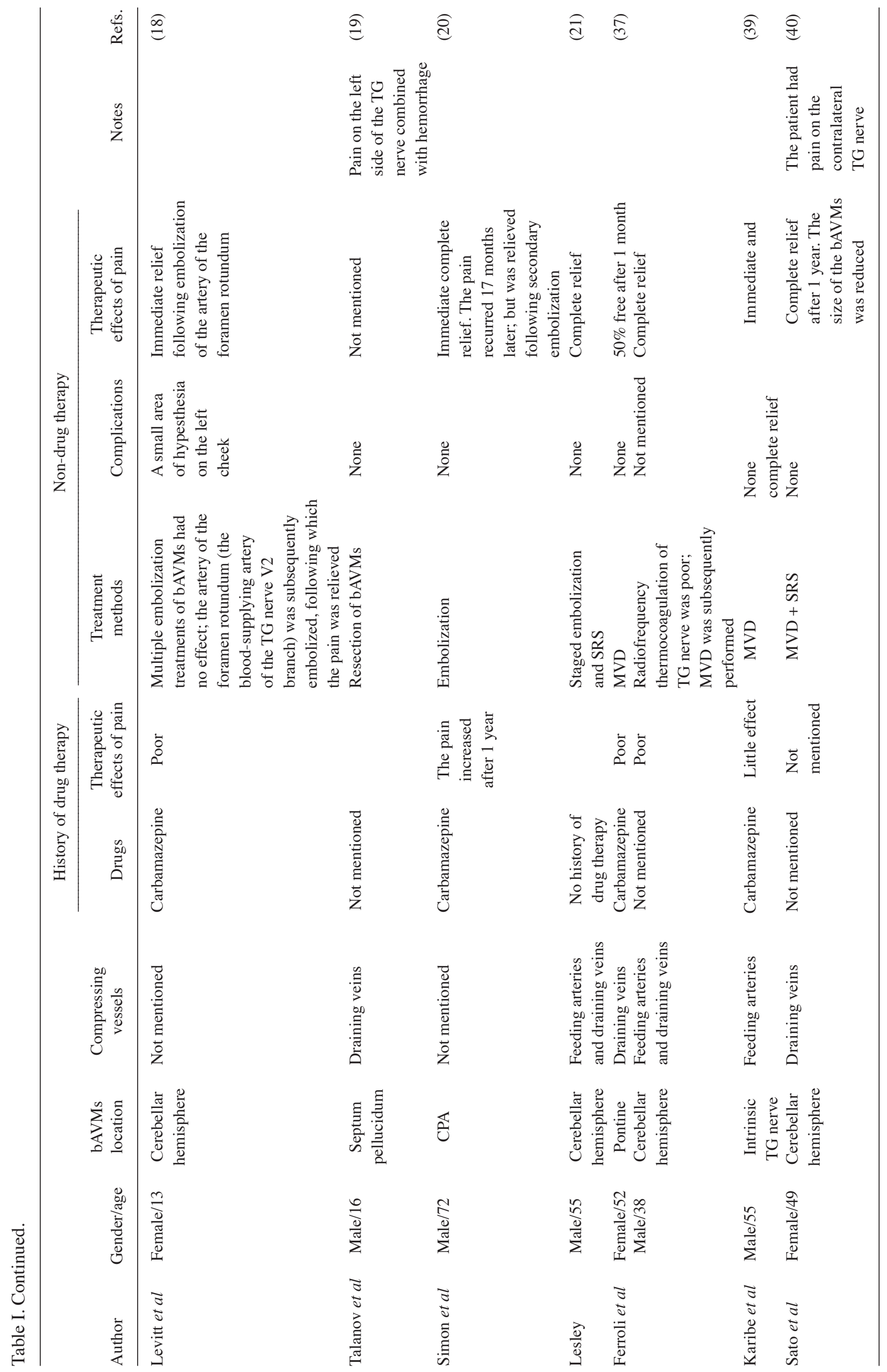




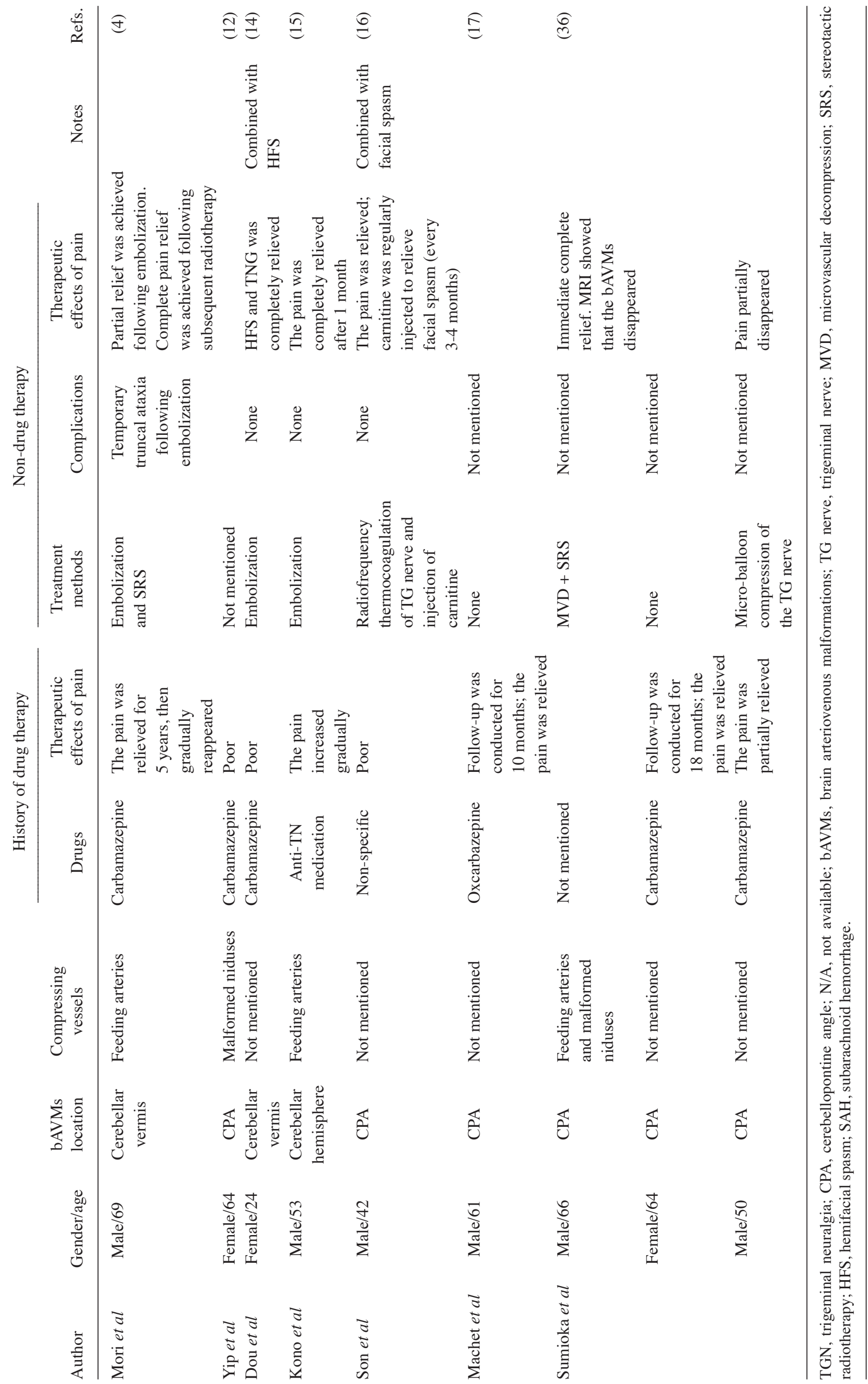


A
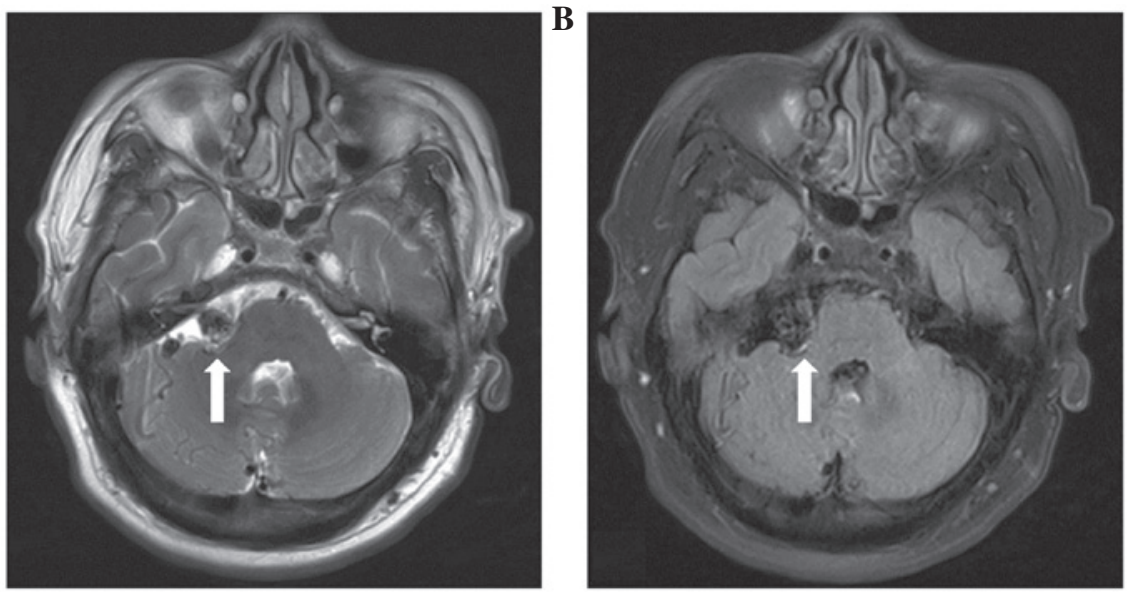

Figure 1. (A) T2-weighted axial MRI and (B) T1-weighted axial MRI showing flow-void signals (arrows) of abnormal vessels in the right cerebellopontine angle prior to the surgical procedure. MRI, magnetic resonance imaging.

A

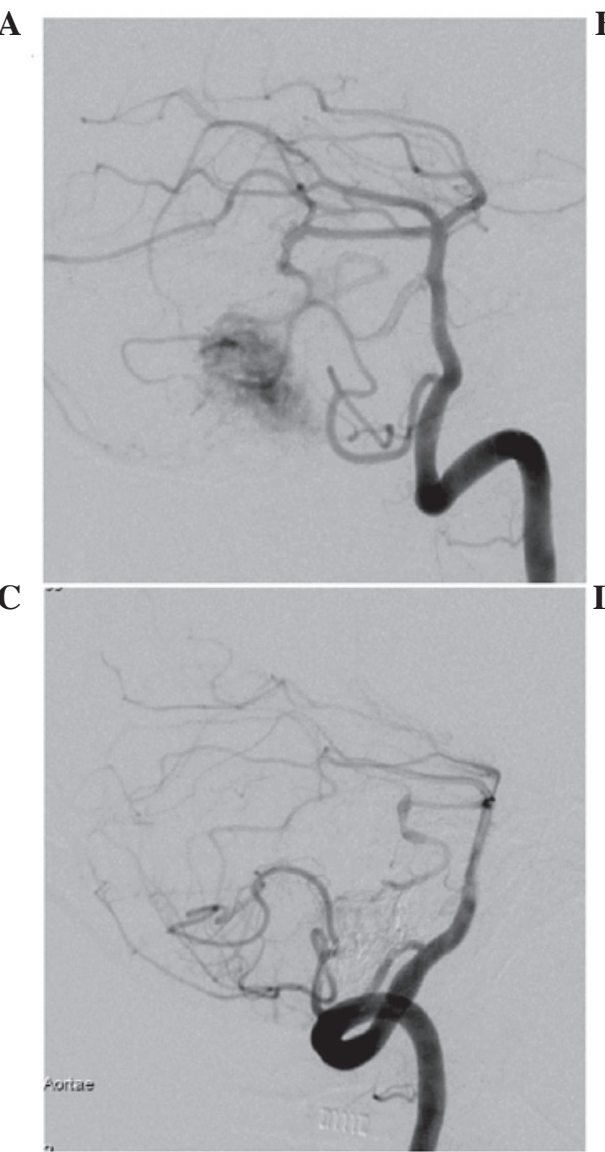

B

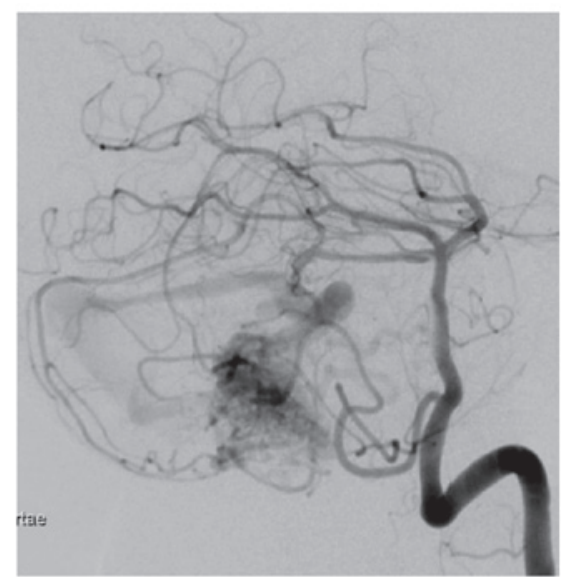

D

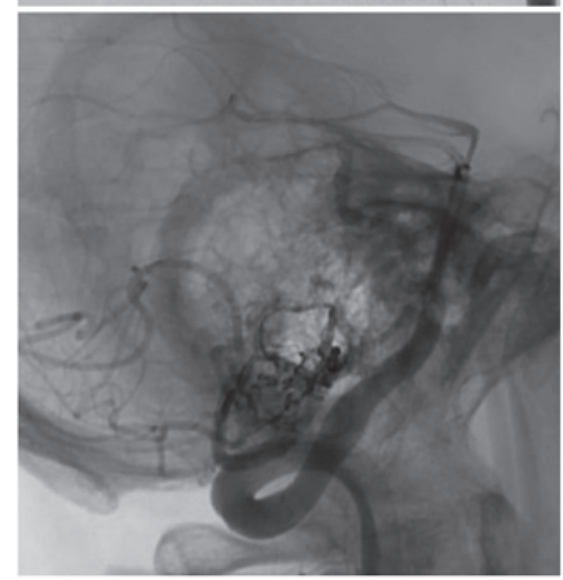

Figure 2. (A and B) Lateral views of DSA prior to embolization shows the bAVMs, which are associated with the right superior cerebellar and the right anterior inferior cerebellar, and draining rapidly into the adjacent venous sinuses on the same side. (C and D) Lateral views of the DSA following embolization show that the majority of the bAVMs were embolized. DSA, digital subtraction angiography; bAVMs, brain arteriovenous malformations.

compression of the contralateral TG nerve REZ by the arterialized draining veins (40). The aforementioned findings also demonstrated that TGN caused by bAVMs is a symptom of neurovascular compression, caused by the pulsatile compression of vessels. Pulsatile compression of the vessels may be caused by the feeding arteries of bAVMs, arterialized draining veins or malformation niduses $(38,40,43)$. Among the 40 patients evaluated $(4,12,14-40)$, there were 27 patients
$(4,12,15,19,21,24-29,31,34-40)$ in whom TGN was demonstrated to originate from vascular compression as determined by angiography or during the surgical procedure. Of these 27 patients, TGN was caused by compression by the feeding arteries of bAVMs $(n=8)(4,15,24,28,29,35,38,39)$, the draining venous system $(n=4)(19,26,37,40)$, malformation niduses $(n=5)$ $(12,27,38)$, simultaneous compression by the feeding arteries and the draining venous system $(n=3)(21,34,38)$, simultaneous 


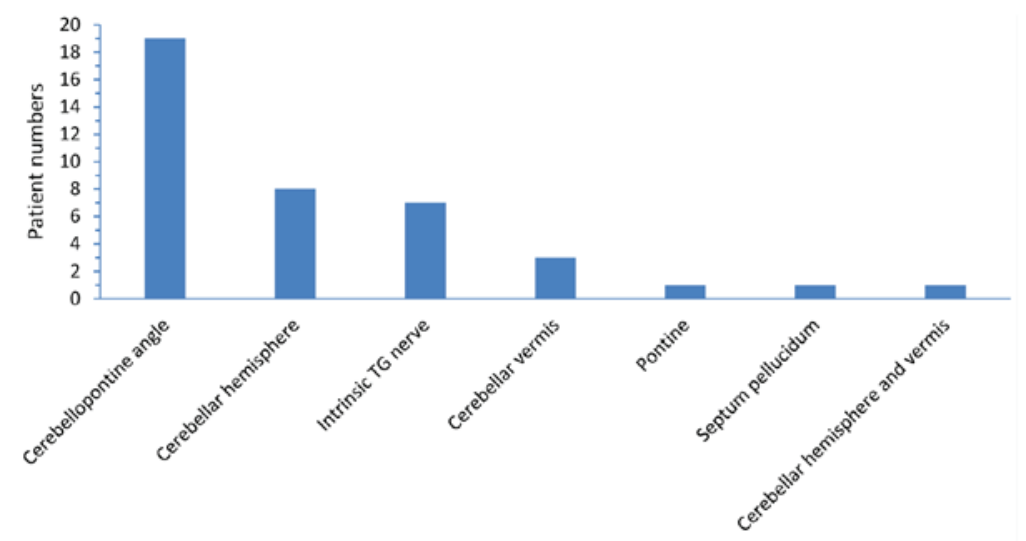

Figure 3. Respective locations of the bAVMs in patients with trigeminal neuralgia caused by bAVMs as reported in previous studies ( $\mathrm{n}=40$ ). bAVMs, brain arteriovenous malformations.

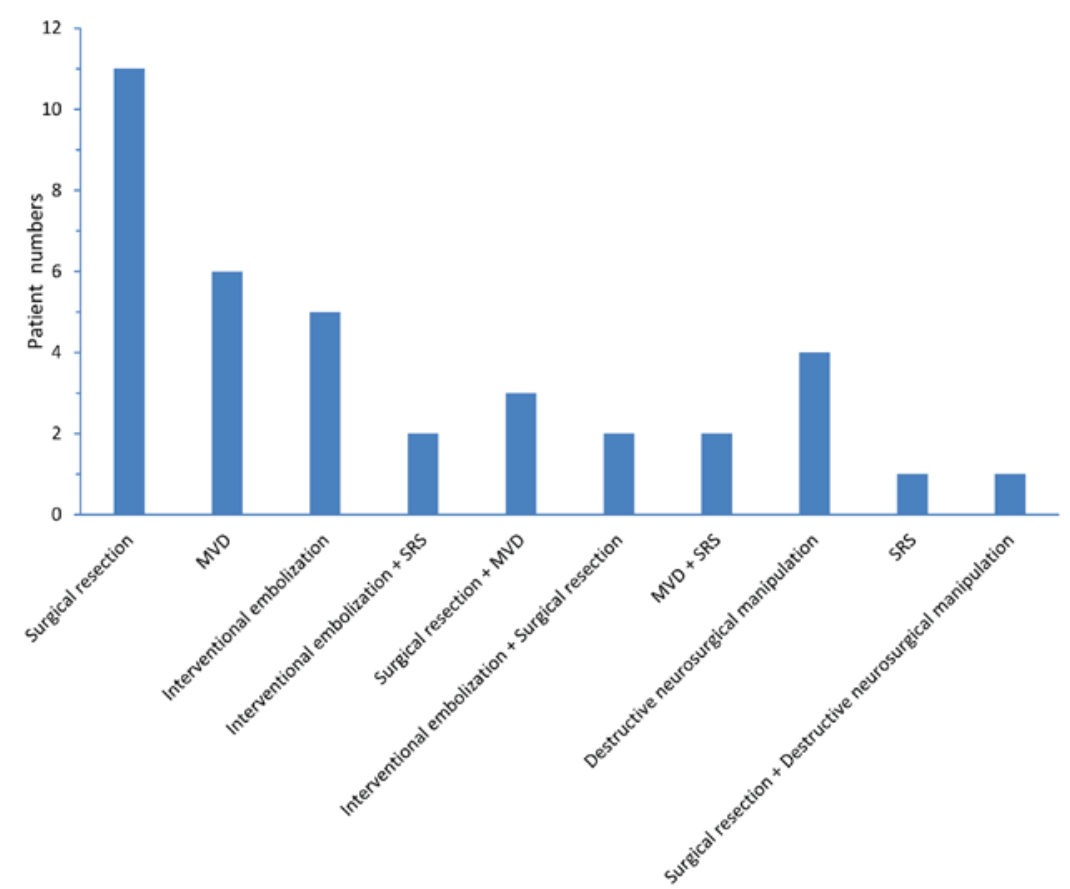

Figure 4. Non-drug treatment methods used for patients with trigeminal neuralgia caused by brain arteriovenous malformations, as reported in previous studies $(\mathrm{n}=37)$. MVD, microvascular decompression; SRS, stereotactic radiotherapy.

compression by the feeding arteries and the malformation niduses $(n=1)(36)$, the simultaneous compression by the draining venous system and the malformation niduses $(n=5)$ $(25,31)$ and simultaneous compression by the feeding arteries, the draining venous system and the malformation niduses $(n=1)(25)$. Since the origin of vascular compression could not be confirmed by image analysis in certain patients, and these patients had also received drug therapy, interventional embolization, and destructive neurosurgical manipulation of the TG nerve, the incidence of TGN originating from vascular compression may be even higher.

Currently, the majority of treatments for TGN caused by bAVMs are determined on an individual basis, and there has been no consistent conclusion as to the optimal treatment for TGN caused by bAVMs (14-16). Drug therapy did not yield an ideal therapeutic effect in the majority of cases, and the patients who received drug therapy predominantly proceeded to seek non-drug treatments $(4,12,14-16,22,23,25,28,30-32)$. Non-drug treatments include surgical resection of bAVMs, MVD, SRS, and destructive neurosurgical manipulation of the TG nerve $(4,16,33,36,37)$. Therefore, when selecting a treatment, it is necessary to comprehensively consider whether the patient has a history of hemorrhage, whether the bAVMs are associated with aneurysms and the origin of the compression of the TG nerve.

Theoretically, complete surgical resection is the ideal therapeutic option, as it eliminates the compression on the TG nerve and avoids the potential risk of hemorrhage of bAVMs, which is particularly important for patients with a history of hemorrhage. In addition, the craniotomy for resection of bAVMs may also confirm whether the feeding arteries and the draining veins compress the TG nerve root; if necessary, MVD can be performed simultaneously (44). Among the 40 patients evaluated $(4,12,14-40), 42.5 \%$ of the patients 
received surgical resection $(n=17)$, and amongst these 17 patie nts $(19,23,25-32,34,35), 11$ patients received surgical resection alone $(19,25,27-30,32), 3$ patients received surgical resection combined with MVD $(25,34,35)$, and 2 patients received surgical resection due to complications following embolization $(23,26)$, and 1 patient received surgical resection due poor effect after destructive neurosurgical manipulation of the TG nerve (31). The pain caused by TGN was immediately relieved in all the patients who received surgical resection. The longest duration of follow-up visits in these previous studies was 109 months (25). According to the long-term follow-up visits reported, relapsed pain was only reported in one patient at a four-year post-surgical follow-up, and this pain was controlled by $400 \mathrm{mg} /$ day carbamazepine (25). The majority of these lesions were located deep in the posterior fossa adjacent to the brain stem and surrounding important nerves $(23,26,27)$. Furthermore, there were numerous complications during surgical resection, particularly for patients with bAVMs in the TG nerve REZ. Edwards et al (25) reported the treatment of five patients with bAVMs in the TG nerve REZ through surgical resection, and following the surgical procedure, four of these patients experienced varying levels of dysesthesia of the TG nerve, and one patient also experienced weakening of the facial neurological function, short-term ataxia and cerebellar hematoma. Although the remission rate of TNG is very high after surgical resection, there are also many complications. Thus it is a necessary to weigh the benefits and risks before the operation.

It is sometimes difficult to perform complete surgical resection of bAVMs, and this procedure may also be unnecessary for some patients. Among the 40 patients $(4,12,14-40)$ with TGN caused by bAVMs, $55 \%$ of patients $(n=22)$ (4,15,19,21,24-26,28,29,33-40) presented with TGN which partially or completely originated from the compression of feeding arteries or the draining veins, which was confirmed by image analysis or during the surgical procedure. Therefore, surgical resection of bAVMs was unnecessary in these patients with TGN with no history of hemorrhage, and MVD surgery would have been sufficient. MVD surgery of the TG nerve is $>90 \%$ effective and is capable of preserving neurological function relatively well; thus, MVD surgery has become the first choice of surgical treatment for primary TGN (45). Furthermore, bAVMs in the CPA, and even malformed niduses in the TG nerve, may not necessarily result in TGN $(39,43,46)$. Therefore, focusing on complete resection of bAVMs as the predominant therapeutic option may cause further complications. The mid-term results of a multicenter, randomized control study which investigated the treatments of unruptured bAVMs demonstrated that the effects of drug therapies are superior to those of invasive therapies when treating unruptured bAVMs (47). Among the 40 patients evaluated in the present review $(4,12,14-40), 27 \%$ of patients $(n=11)$ received MVD surgery, with the longest duration of follow-up being 18 months (36). A $100 \%$ overall effective rate was achieved and no surgery-associated complications occurred (34-40).

Interventional embolization can be used to reduce the size of bAVMs by reducing blood supply to bAVMs $(4,21,23,26)$. This procedure is predominantly performed to surgically treat bAVMs or as an adjuvant therapy prior to $\operatorname{SRS}(4,21,23,26)$. However, when treating TGN caused by bAVMs, TGN may be effectively relieved following partial embolization of bAVMs due to the change in hemodynamics, which also demonstrates that TGN caused by bAVMs is a symptom of neurovascular compression (20). Due to these characteristics, interventional embolization was performed on the present patient and TGN was completely relieved following successful embolization of the majority of the bAVMs. Post-embolization follow-ups were conducted for two years, and the therapeutic effect of this treatment option remained satisfactory. Among the 40 patients $(4,12,14-40)$ evaluated in the present review, $22.5 \%$ of patients $(n=9)(4,14,15,18,20,21,23,24,26)$ underwent interventional embolization and experienced significantly relieved TGN following embolization. Among these 9 patients, 5 patients received an interventional embolization treatment alone $(14,15,18,20,24), 2$ patients received subsequent SRS $(4,21)$ and 2 patients received secondary surgical resection $(23,26)$. The longest duration of follow-up visits for patients who underwent interventional embolization was six years (4). One patient reported relapsed pain 17 months post-embolization and the pain was subsequently relieved following a secondary embolization (4). The pain relief may be associated with the blocking of the pulsatile compression of the feeding arteries of the bAVMs, the arterialized draining veins or the malformed niduses that compressed the TG nerve following embolization, which appears to have a similar mechanism to the MVD of the TG nerve. However, caution should be taken with this therapeutic option as interventional embolization and the change in hemodynamics following embolization may induce ischemia, hemorrhage or embolization of the venous system $(4,23,26)$. Among the aforementioned eight patients who received embolization, one patient experienced transient ataxia (4), one patient received secondary surgical resection of bAVMs due to subarachnoid hemorrhage and hydrocephalus following the embolization (23), and one patient suffered from severe paresis and sensory disturbance due to the dilated draining veins following embolization, which required surgical resection (26). Furthermore, since the rate of complete embolization is restricted when treating bAVMs with interventional therapy alone, and post-embolization recanalization is relatively common, embolization alone may not result in a long-term therapeutic effect. Simon et al (20) reported the case of a patient with TGN caused by bAVMs whose pain was relieved following embolization; however, the pain recurred 17 months later. It has also been demonstrated that only short-term pain relief was achieved following multiple embolization procedures (18), which may be associated with the recanalization of bAVMs.

SRS is often performed following partial embolization or combined with other treatment methods $(4,21,36,40)$. Among the 40 patients evaluated in the present review (4,12,14-40), there was only one report (22) of the achievement of an effective therapeutic effect of SRS alone. In this case, the bAVMs were located inside the TG nerve and the size of the malformation niduses was $\sim 1.2 \times 0.8 \times 0.9 \mathrm{~cm}$; the TGN was completely relieved following 13 months of SRS (22). Notably, Levitt et al (18) treated a patient with TGN by embolizing the supplying artery of the V2 branch of the TG nerve, an artery of the foramen rotundum, which induced sustained relief from TGN, but resulted in hypoesthesia of the cheeks on the same side. Previous studies have also reported other 
palliative destructive neurosurgical techniques of the TG nerve root $(16,17,33,38)$, including radiofrequency thermocoagulation, glycerol rhizolysis, balloon compression and resection of the TG nerve; however, these palliative therapies may result in complications, such as facial numbness and weakened corneal reflexes $(16,17,33,38)$. Furthermore, the therapeutic effects of these palliative therapies remain uncertain, and TGN has a relatively high recurrence rate when treated with these therapeutic strategies $(16,17,33,38)$.

In conclusion, surgical resection of the malformed vessels is an ideal therapeutic option in theory, as this approach relieves TGN and eliminates the risk of hemorrhage of bAVMs; whereas MVD surgery is a recommended treatment for patients who cannot receive surgical resection of bAVMs. Good therapeutic outcomes have also been achieved with interventional embolization in certain patients, including the patient described in the present case study. Destructive neurosurgical manipulation of the TG nerve may result in facial numbness and weakened corneal reflexes, and has a high recurrence rate; therefore, this approach is only recommended as an alternative for elderly surgery-intolerant patients.

\section{References}

1. Love S and Coakham HB: Trigeminal neuralgia: Pathology and pathogenesis. Brain 124: 2347-2360, 2001.

2. Cruccu G, Gronseth G, Alksne J, Argoff C, Brainin M, Burchiel K, Nurmikko T and Zakrzewska JM; American Academy of Neurology Society; European Federation of Neurological Society: AAN-EFNS guidelines on trigeminal neuralgia management. Eur J Neurol 15: 1013-1028, 2008.

3. Headache Classification Committee of the International Headache Society (IHS): The international classification of headache disorders, 3 rd edition (beta version). Cephalalgia 33: 629-808, 2013.

4. Mori Y, Kobayashi T, Miyachi S, Hashizume C, Tsugawa T and Shibamoto Y: Trigeminal neuralgia caused by nerve compression by dilated superior cerebellar artery associated with cerebellar arteriovenous malformation: Case report. Neurol Med Chir (Tokyo) 54: 236-241, 2014.

5. Ishikawa M, Nishi S, Aoki T, Takase T, Wada E, Ohwaki H, Katsuki $\mathrm{T}$ and Fukuda H: Operative findings in cases of trigeminal neuralgia without vascular compression: Proposal of a different mechanism. J Clin Neurosci 9: 200-204, 2002.

6. McLaughlin MR, Jannetta PJ, Clyde BL, Subach BR, Comey $\mathrm{CH}$ and Resnick DK: Microvascular decompression of cranial nerves: Lessons learned after 4400 operations. J Neurosurg 90: 1-8, 1999.

7. Mast H, Young WL, Koennecke H-C, et al: Risk of spontaneous haemorrhage after diagnosis of cerebral arteriovenous malformation. The Lancet 350: 1065-1068, 1997.

8. Nataraj A, Mohamed MB, Gholkar A, Vivar R, Watkins L, Aspoas R, Gregson B, Mitchell P and Mendelow AD: Multimodality treatment of cerebral arteriovenous malformations. World Neurosurg 82: 149-159, 2014.

9. Whitehead KJ, Smith MC and Li DY: Arteriovenous malformations and other vascular malformation syndromes. Cold Spring Harb Perspect Med 3: a006635, 2013.

10. Rodríguez-Hernández $\mathrm{A}, \mathrm{Kim} \mathrm{H}$, Pourmohamad $\mathrm{T}$, Young WL and Lawton MT; University of California, San Francisco Arteriovenous Malformation Study Project: Cerebellar arteriovenous malformations: Anatomic subtypes, surgical results, and increased predictive accuracy of the supplementary grading system. Neurosurgery 71: 1111-1124, 2012.

11. Mast H, Young WL, Koennecke HC, Sciacca RR, Osipov A, Pile-Spellman J, Hacein-Bey L, Duong H, Stein BM and Mohr JP: Risk of spontaneous haemorrhage after diagnosis of cerebral arteriovenous malformation. Lancet 350: 1065-1068, 1997.
12. Yip V, Michael BD, Nahser HC and Smith D: Arteriovenous malformation: a rare cause of trigeminal neuralgia identified by magnetic resonance imaging with constructive interference in steady state sequences. QJM : monthly journal of the Association of Physicians 105: 895-898, 2012.

13. Seldinger SI: Catheter replacement of the needle in percutaneous arteriography: a new technique. Acta Radiol 39: 368-376, 1953.

14. Dou NN, Hua XM, Zhong J and Li ST: A successful treatment of coexistent hemifacial spasm and trigeminal neuralgia caused by a huge cerebral arteriovenous malformation: A case report. J Craniofac Surg 25: 907-910, 2014.

15. Kono K, Matsuda Y and Terada T: Resolution of trigeminal neuralgia following minimal coil embolization of a primitive trigeminal artery associated with a cerebellar arteriovenous malformation. Acta Neurochir (Wien) 155: 1699-1701, 2013.

16. Son BC, Kim DR, Sung JH and Lee SW: Painful tic convulsif caused by an arteriovenous malformation. Clin Neuroradiol 22: 365-369, 2012.

17. Machet A, Aggour M, Estrade L, Chays A and Pierot L: Trigeminal neuralgia related to arteriovenous malformation of the posterior fossa: Three case reports and a review of the literature. J Neuroradiol 39: 64-69, 2012.

18. Levitt MR, Ramanathan D, Vaidya SS, Hallam DK and Ghodke BV: Endovascular palliation of AVM-associated intractable trigeminal neuralgia via embolization of the artery of the foramen rotundum. Pain Med 12: 1824-1830, 2011

19. Talanov AB, Filatov Iu M, Eliava ShSh, Novikov AE and Kulishova IaG: Arteriovenous malformation of septum pellucidum in combination with persistent trigeminal neuralgia. Zh Vopr Neirokhir Im N N Burdenko 50-53; discusion 53-54, 2009 (In Russian).

20. Simon SD, Yao TL, Rosenbaum BP, Reig A and Mericle RA: Resolution of trigeminal neuralgia after palliative embolization of a cerebellopontine angle arteriovenous malformation. Cent Eur Neurosurg 70: 161-163, 2009.

21. Lesley WS: Resolution of trigeminal neuralgia following cerebellar AVM embolization with Onyx. Cephalalgia 29: 980-985, 2009.

22. Anderson WS, Wang PP and Rigamonti D: Case of microarteriovenous malformation-induced trigeminal neuralgia treated with radiosurgery. J Headache Pain 7: 217-221, 2006.

23. Wanke I, Dietrich U, Oppel F and Puchner MJ: Endovascular treatment of trigeminal neuralgia caused by arteriovenous malformation: Is surgery really necessary? Zentralbl Neurochir 66: 213-216, 2005.

24. Athanasiou TC, Nair S, Coakham HB and Lewis TT: Arteriovenous malformation presenting with trigeminal neuralgia and treated with endovascular coiling. Neurol India 53: 247-248, 2005.

25. Edwards RJ, Clarke Y, Renowden SA and Coakham HB: Trigeminal neuralgia caused by microarteriovenous malformations of the trigeminal nerve root entry zone: Symptomatic relief following complete excision of the lesion with nerve root preservation. J Neurosurg 97: 874-880, 2002.

26. Mineura K, Sasajima H, Itoh Y, Kowada M, Tomura N and Goto K: Development of a huge varix following endovascular embolization for cerebellar arteriovenous malformation. A case report. Acta Radiol 39: 189-192, 1998.

27. Nomura T, Ikezaki K, Matsushima T and Fukui M: Trigeminal neuralgia: Differentiation between intracranial mass lesions and ordinary vascular compression as causative lesions. Neurosurg Rev 17: 51-57, 1994.

28. Mendelowitsch A, Radue EW and Gratzl O: Aneurysm, arteriovenous malformation and arteriovenous fistula in posterior fossa compression syndrome. Eur Neurol 30: 338-342, 1990.

29. Kikuchi K, Kamisato N, Sasanuma J, Watanabe K and Kowada M: Trigeminal neuralgia associated with posterior fossa arteriovenous malformation and aneurysm fed by the same artery. Case report. Neurol Med Chir (Tokyo) 30: 918-921, 1990 (In Japanese)

30. Figueiredo PC, Brock M, De Oliveira AM Júnior and Prill A: Arteriovenous malformation in the cerebellopontine angle presenting as trigeminal neuralgia. Arq Neuropsiquiatr 47: 61-71, 1989.

31. Kawano H, Hayashi M, Kobayashi H, Tsuji T, Kabuto M and Kubota T: Gas CT cisternography of trigeminal neuralgia caused by AVM of the cerebellopontine angle. AJNR Am J Neuroradiol 8: 161-162, 1987.

32. Kawano H, Kobayashi H, Hayashi M, Tsuji T, Kabuto M and Nozaki J: Trigeminal neuralgia caused by arteriovenous malformation of the cerebellopontine angle-report of two cases. No to Shinkei 36: 1175-1179, 1984 (In Japanese). 
33. Johnson MC and Salmon JH: Arteriovenous malformation presenting as trigeminal neuralgia. Case report. J Neurosurg 29: 287-289, 1968

34. Niwa J, Yamamura A and Hashi K: Cerebellar arteriovenous malformation presenting as trigeminal neuralgia. No Shinkei Geka 16: 753-756, 1988 (In Japanese).

35. Nishizawa Y, Tsuiki K, Miura K, Murakami M, Kirikae M, Hakozaki S, Saiki I and Kanaya H: Multiple arteriovenous malformations of left parietal lobe and left cerebellar hemisphere with symptomatic trigeminal neuralgia: A case report. No Shinkei Geka 16 (Suppl 5): S625-S630, 1988 (In Japanese).

36. Sumioka S, Kondo A, Tanabe $H$ and Yasuda S: Intrinsic arteriovenous malformation embedded in the trigeminal nerve of a patient with trigeminal neuralgia. Neurol Med Chir (Tokyo) 51: 639-641, 2011.

37. Ferroli P, Acerbi F, Broggi $M$ and Broggi G: Arteriovenous micromalformation of the trigeminal root: Intraoperative diagnosis with indocyanine green videoangiography: Case report. Neurosurgery 67 (3 Suppl Operative): onsE309-onsE310; discussion onsE310, 2010 .

38. García-Pastor C, López-González F, Revuelta R and Nathal E: Trigeminal neuralgia secondary to arteriovenous malformations of the posterior fossa. Surg Neurol 66: 207-211; discussion 211 2006.

39. Karibe H, Shirane R, Jokura H and Yoshimoto T: Intrinsic arteriovenous malformation of the trigeminal nerve in a patient with trigeminal neuralgia: Case report. Neurosurgery 55: 1433, 2004.

40. Sato K, Jokura H, Shirane R, Akabane T, Karibe H and Yoshimoto T: Trigeminal neuralgia associated with contralateral cerebellar arteriovenous malformation. Case illustration. J Neurosurg 98: 1318, 2003.
41. Garcia M, Naraghi R, Zumbrunn T, Rösch J, Hastreiter P and Dörfler A: High-resolution 3D-constructive interference in steady-state MR imaging and 3D time-of-flight MR angiography in neurovascular compression: A comparison between $3 \mathrm{~T}$ and 1.5T. AJNR Am J Neuroradiol 33: 1251-1256, 2012.

42. Linn J, Moriggl B, Schwarz F, Naidich TP, Schmid UD, Wiesmann M, Bruckmann H and Yousry I: Cisternal segments of the glossopharyngeal, vagus, and accessory nerves: Detailed magnetic resonance imaging-demonstrated anatomy and neurovascular relationships. J Neurosurg 110: 1026-1041, 2009.

43. Krischek B, Yamaguchi S, Sure U, Benes L, Bien S and Bertalanffy $\mathrm{H}$ : Arteriovenous malformation surrounding the trigeminal nerve-case report. Neurol Med Chir (Tokyo) 44: 68-71, 2004.

44. Abla AA, Nelson J, Rutledge WC, Young WL, Kim H and Lawton MT: The natural history of AVM hemorrhage in the posterior fossa: Comparison of hematoma volumes and neurological outcomes in patients with ruptured infra- and supratentorial AVMs. Neurosurg Focus 37: E6, 2014.

45. Zakrzewska JM and Linskey ME: Trigeminal neuralgia. Clin Evid (Online) 10: 1207, 2014.

46. Maher CO, Atkinson JL and Lane JI: Arteriovenous malformation in the trigeminal nerve. Case report. J Neurosurg 98: 908-912, 2003.

47. Mohr JP, Parides MK, Stapf C, Moquete E, Moy CS, Overbey JR, Al-Shahi Salman R, Vicaut E, Young WL, Houdart E, et al: Medical management with or without interventional therapy for unruptured brain arteriovenous malformations (ARUBA): A multicentre, non-blinded, randomised trial. Lancet 383: 614-621, 2014. 